\title{
Planejamento de Caminhos Seguros e Suaves Baseados em Espuma Probabilística para Sistemas Robóticos Autônomos
}

\author{
Luís B. P. Nascimento e Pablo J. Alsina (Orientador)
}

\author{
${ }^{1}$ Programa de Pós-Graduação em Engenharia Elétrica e de Computação \\ Universidade Federal do Rio Grande do Norte - Natal - RN - Brasil \\ lbrunoeufrn.edu.br e pabloddca.ufrn.br
}

\begin{abstract}
Resumo. O Método da Espuma Probabilística (PFM) é um planejador capaz de gerar caminhos que garantem uma região segura para manobra. Essa característica é ideal para aplicações em robótica assistiva que requerem caminhos com um maior grau de segurança. Entretanto, o PFM necessita da representação explícita dos obstáculos no espaço de configurações, o que inviabiliza, na prática, sua aplicação para a maioria dos problemas robóticos. Dessa forma, este trabalho apresenta uma nova abordagem para que o PFM não compute a região de obstáculos. Além disso, são apresentadas estratégias diminuir o comprimento e aumentar a suavidade do caminho planejado, mantendo a principal característica da abordagem que é a segurança. Experimentos simulados utilizando as técnicas propostas foram realizados para um exoesqueleto para membros inferiores e para um andador inteligente, ambos robôs assistivos.
\end{abstract}

Tese de Doutorado submetida ao Graduate Works Contest in Robotics (CTDR). Data de defesa do trabalho 30 de agosto de 2021.

\section{Introdução}

Planejar um caminho livre de obstáculos para um robô navegar evitando colisões é uma questão fundamental da robótica autônoma. Em geral, os planejadores focam na obtenção de caminhos ótimos ou na redução do tempo da busca [Volna and Kotyrba 2018]. Entretanto, também é essencial que eles garantam um grau de segurança para evitar possíveis colisões no ambiente [Plaku et al. 2018]. Dessa forma, é de grande importância planejar caminhos suficientemente afastados dos obstáculos [Berglund et al. 2009].

O Método da Espuma Probabilística (Probabilistic Foam Method - PFM) [Silveira and Alsina 2016], é um planejador que garante uma região livre de obstáculos para manobrabilidade segura por meio da bolha, uma estrutura volumétrica, que representa um sub-conjunto do espaço livre $\mathcal{C}_{\text {livre }}$. Um conjunto de bolhas conectadas são expandidas iterativamente, formando a estrutura Espuma Probabilística, que se propaga partindo da configuração inicial até a final, realizando uma cobertura aproximada de $\mathcal{C}_{\text {livre }}$.

O PFM é ideal para aplicações na robótica assistiva, pois obtém caminhos com a garantia de uma região segura. Entretanto, é computacionalmente inviável, do ponto de vista prático, para problemas várias dimensões, devido a computação da região de obstáculos, algo que não é trivial. Além disso, os caminhos gerados não são suaves.

Dessa forma, esse trabalho apresenta uma estratégia para a computação das bolhas para evitar o cálculo da região C-obstáculos. Além disso, será apresentada a 
implementação de estratégias para a suavização dos caminhos obtidos a partir de métodos baseados em espuma probabilística, garantindo a segurança imposta pelas bolhas.

Foi utilizado como estudo de caso o exoesqueleto Ortholeg [Araujo et al. 2015] e o andador Cloud Walker [Scheidegger et al. 2019]. O uso desses dispositivos se justifica pois eles interagem diretamente com os seres humanos para prover a mobilidade assistida, tornando assim necessária a garantia de mais segurança e confiabilidade durante os movimentos. Os projetos Ortholeg e Cloud Walker são ilustrados na Figura 1.

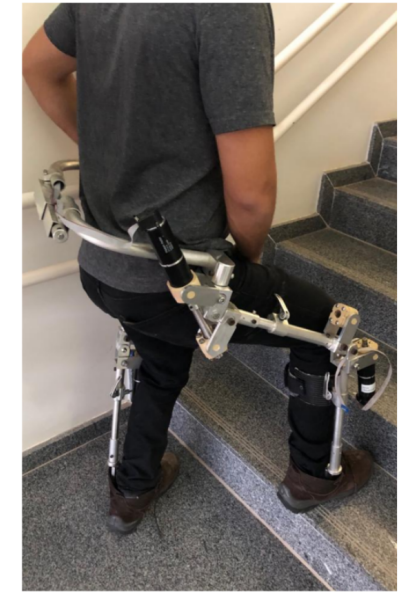

(a)

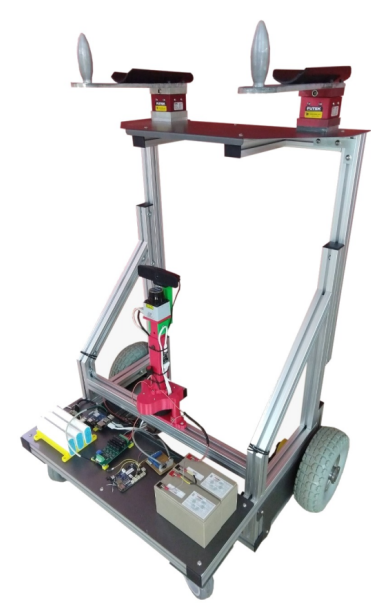

(b)

Figura 1. (a) $\mathrm{O}$ exoesqueleto Ortholeg. (b) $\mathrm{O}$ andador Cloud Walker

\section{Método da Espuma Probabilística}

Seja $\mathcal{C}$ o espaço $n$-dimensional de todas as possíveis configurações $q$ que um robô pode assumir, $\mathcal{C}_{\text {obs }}$ a região de obstáculos em $\mathcal{C}$, e $\mathcal{C}_{\text {livre }}$ a região livre, o espaço de configurações é definido como $\mathcal{C}=\mathcal{C}_{\text {livre }} \cup \mathcal{C}_{\text {obs }}$.

Definicão 2.1 (A bolha $b$ ) A bolha b é uma n-bola computada na região $\mathcal{C}_{\text {livre, }}$ com raio $r$, centrada na configuração $q_{c}$, definida por:

$$
b=b\left(q_{c}, r\right)=\left\{q: d\left(q, q_{c}\right)<r\right\}
$$

tal que d $\left(q, q_{c}\right)$ é a distância entre a configuração $q_{c}$ e uma configuração q qualquer, de acordo com a métrica utilizada no espaço $\mathcal{C}$.

$\mathrm{O}$ raio da bolha é calculado pela menor distancia entre $q_{c}$ e o obstáculo mais próximo. Assim, ela representa um conjunto de configurações que um robô pode assumir sem colidir com os obstáculos. Uma bolha mãe $b_{\text {mae }}$ permite a expansão de novas bolhas filhas $b_{\text {filha }}$ sobre sua superfície. As bolhas conectadas com grau de parentesco direto formam uma região de interseção chamada Escotilha.

Definicão 2.2 (A região da Escotilha) A escotilha é uma região não vazia formada pela interseção entre duas bolhas sobrepostas e conectadas, seguindo a relação de parentesco mãe-filha (uma bolha $b_{\text {filha }}$ conectada sobre a superfície de uma bolha $b_{\text {mae }}$ ).

Definicão 2.3 (A região de passagem ou "Portal") A navegabilidade segura entre duas bolhas adjacentes conectadas (com grau de parentesco direto) é possível devido a uma região chamada Portal. Essa região é localizada dentro da escotilha e é definida como uma (n-1)-bola, delimitada por uma hiperesfera de dimensão $n-2$, isto é, $(n-2)$-esfera. 
Uma bolha mãe pode gerar um conjunto de bolhas filhas sobre a sua superfície, e essas filhas também podem gerar novas bolhas, que dentro de um processo iterativo, vai formar uma estrutura chamada de espuma probabilística.

Definicão 2.4 (A espuma probabilística $E$ ) $O$ conjunto $E$ denominado espuma probabilística é uma coleção de bolhas $b_{i}$ sobrepostas. A espuma é construída incrementalmente por meio da expansão de bolhas filhas sobre a superfície de bolhas mães.

\subsection{A propagação da espuma probabilística}

A espuma probabilística se propaga em $\mathcal{C}_{\text {livre }}$ com bolhas filhas que são expandidas na superfície das bolhas mães de gerações anteriores. A Figura 2 ilustra o processo de propagação da espuma para um ambiente mapeado para um C-space de duas dimensões.

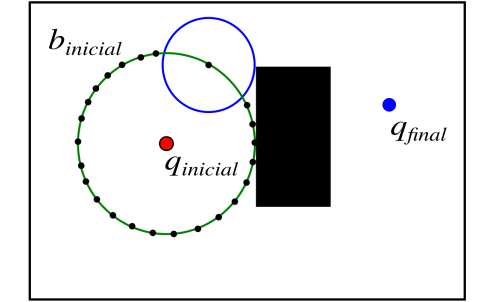

(a)

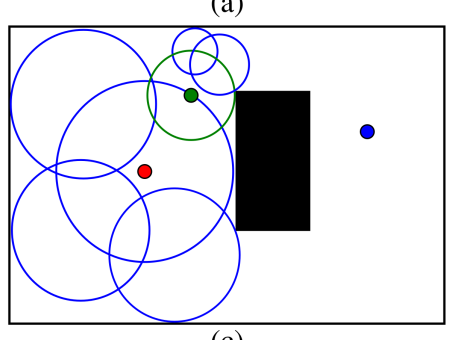

(c)

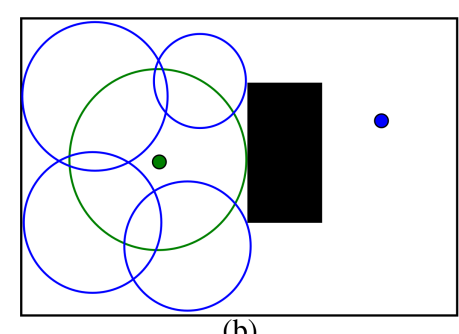

(b)

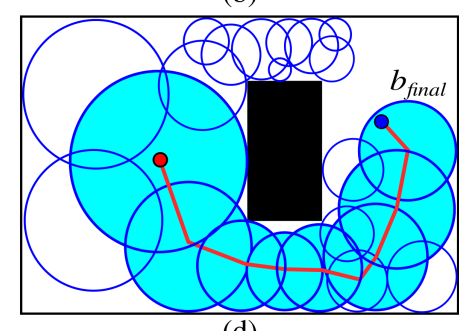

(d)

Figura 2. (a) Configurações aleatórias na superfície da bolha. (b) Quatro bolhas filhas são expandidas. (c) Seleção da nova bolha mãe. (d) A espuma foi propagada e um caminho foi extraído.

Na Figura 2a é possível observar um conjunto de pontos sobre a superfície de $b_{\text {inicial }}$. Esses pontos representam os centros de possíveis bolhas filhas que podem ser expandidas sobre essa bolha mãe (representada com superfície verde). Sendo $n$ a dimensão de $\mathcal{C}$ e $r$ o raio da bolha mãe, o seu número máximo de bolhas filhas $N$ é definido por:

$$
N=K\left(\left\lfloor\frac{r}{r_{\min }}\right\rfloor\right)^{n-1}
$$

O parâmetro $r_{\text {min }}$ indica o menor raio admissível e a constante $K$ define o número máximo de bolhas filhas que as bolhas com raio $r_{\min }$ podem gerar, e é definido em função da dimensão do espaço [Nascimento et al. 2021].

As configurações amostradas em regiões exploradas por outras bolhas são descartadas (Figura 2a). Além disso, a nova bolha é descartada se possuir raio $r<r_{\text {min }}$. As bolhas filhas são enfileiradas, mantendo a ordem para as novas bolhas mães serem selecionadas, como ilustrado na Figura 2c. A espuma se propaga até que uma das bolhas filhas englobe a configuração $q_{\text {final }}$, e assim, o algoritmo finaliza. Uma estrutura chamada Rosário pode ser encontrada (Figura 2d), e caminhos factíveis podem ser extraídos dela. 
Definicão 2.5 (Rosário $\mathcal{R}$ ) Por meio de uma busca descendente pela espuma partindo de $b_{\text {final }}$ até $b_{\text {inicial, }}$, seguindo o grau de parentesco das bolhas $b_{i}\{i \in \mathbb{N} / 1 \leqslant i \leqslant k\}$, é possível encontrar um rosário $\mathcal{R}$, constituído por uma sequencia de $k$ bolhas sobrepostas, partindo da bolha $b_{\text {final }}$ até a bolha $b_{\text {inicial. }}$ Um rosário $\mathcal{R}$ é expresso como:

$$
\mathcal{R}=\left\{b_{1}, b_{2}, \ldots, b_{k-1}, b_{k}\right\}
$$

onde $b_{1}=b_{\text {final }}, b_{k}=b_{\text {inicial }}$ e a bolha $b_{i}$ é mãe da bolha $b_{i-1}$, para $i=2,3, \ldots, k-1, k$.

\section{Bolhas usando Métricas no Espaço de Trabalho}

O conceito "bubbles offree space" [Quinlan 1995], define uma região em $\mathcal{C}_{\text {livre }}$ com base na distância entre o robô e os obstáculos no espaço de trabalho, como ilustra a Figura 3.

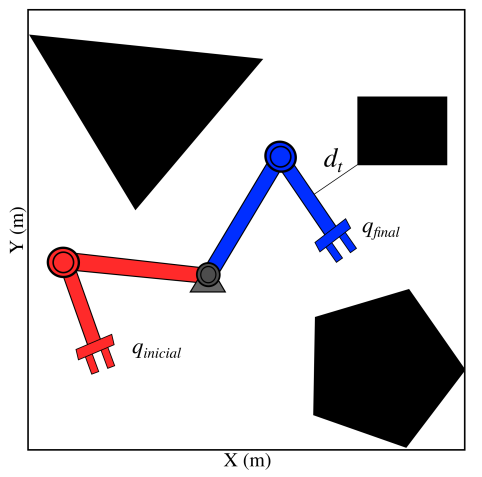

(a)

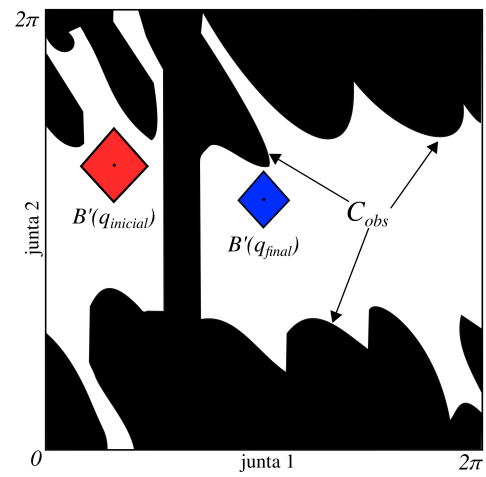

(b)

Figura 3. (a) Manipulador no espaço de trabalho assumindo as configurações $q_{\text {inicial }}$ e $q_{\text {final }}$. (b) Bolhas $B^{\prime}$ no espaço de configurações correspondente às configurações do robô no espaço de trabalho.

Conforme [Quinlan 1995], considerando um robô com $n$ juntas rotacionais, e $d_{t}$ a menor distância entre o robô e os obstáculos no espaço de trabalho $\mathcal{W}$. Se o robô parte de $q$ até uma configuração arbitrária $a$, uma região $B^{\prime}(q)$ pode ser definida como:

$$
B^{\prime}=B^{\prime}(q)=\left\{a: \sum_{i=1}^{n} r_{i}\left|a_{i}-q_{i}\right| \leqslant d_{t}\right\}
$$

Para qualquer movimento partindo de $q$ até qualquer ponto dentro da região $B^{\prime}$, é garantido que nenhum ponto da cadeia cinemática se moverá mais que $d_{t}$. Sendo assim, nenhuma colisão irá ocorrer. A região $B^{\prime}(q)$, resultante no espaço de configuração, será um hiperoctaedro ( $n$-diamante), como é ilustrado na Figura 3, para o caso bidimensional.

Para robôs com rodas de acionamento diferencial, a configuração $q$ do robô é dada por $q=\left[\begin{array}{lll}q_{x} & q_{y} & q_{\theta}\end{array}\right]^{T}$, sendo $q_{x}$ e $q_{y}$ representando a posição cartesiana do robô e $q_{\theta}$ representando seu ângulo de orientação. Considerando que o robô móvel navegue partindo de $q$ até uma configuração arbitrária $q^{\prime}$, realizando um movimento de translação, seguido de um movimento de rotação.

Sendo $d_{t}$, a menor distância entre o limite externo do robô e os obstáculos em $\mathcal{W}$, se a distancia navegada pelo robô for menor que $d_{t}$, nenhuma colisão ocorrerá. Dessa 
forma, a região $B^{\prime}$ para um robô sobre rodas de acionamento diferencial é definida como:

$$
B^{\prime}=B^{\prime}(q)=\left\{q^{\prime}: \sqrt{\left(q_{x}-q_{x}^{\prime}\right)^{2}+\left(q_{y}-q_{y}^{\prime}\right)^{2}}+r_{\text {robo }}\left|q_{\theta}-q_{\theta}^{\prime}\right| \leqslant d_{t}\right\}
$$

Portanto, a região $B^{\prime}$ limita um conjunto de configurações $q^{\prime}$, sendo que a distância navegada pelo robô entre $q$ e $q^{\prime}$ é menor que $d_{t}$. A região $B^{\prime}$, descrita na equação 5 , apresenta a forma de um cone duplo, como pode ser observado na Figura 4.

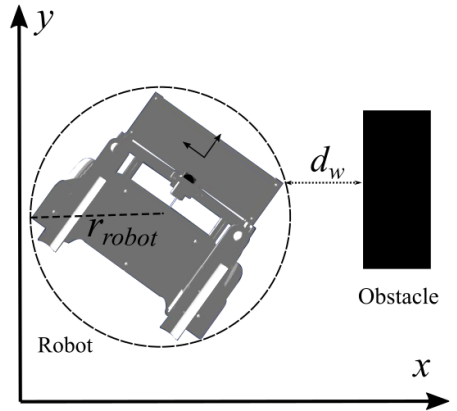

(a)

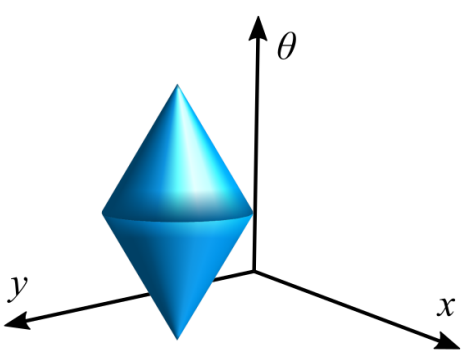

(b)

Figura 4. (a) Modelagem da bolha para o CloudWalker no espaço de trabalho. (b) Bolha no espaço de configurações.

Dessa forma, através da informação da distância entre o robô e o obstáculo no espaço de trabalho, é possível computar as regiões $B^{\prime}$ no espaço de configurações, limitando o movimento do robô dentro de uma região segura.

\subsection{Nova bolha para a Espuma Probabilística}

Como foi mostrado anteriormente, o método Espuma Probabilística trabalha com regiões com formato hiperesférico. Para garantir o uso das regiões modeladas em [Quinlan 1995], calcularemos uma nova bolha $B$ hiperesférica dentro das regiões diamante e cone, denotadas por $B^{\prime}$. Assim, a nova bolha $B$ será uma $(n)$-bola inscrita na região $B^{\prime}$.

Considerando um manipulador com três juntas rotacionais e um robô diferencial, a Figura 5 ilustra a região limitada pela nova bolha $B(q)$.

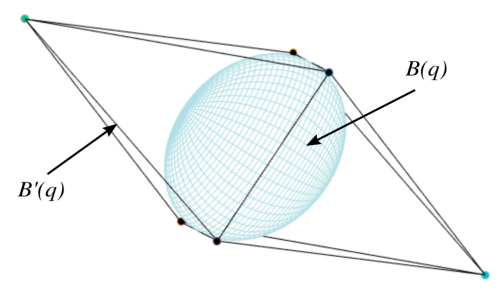

(a)

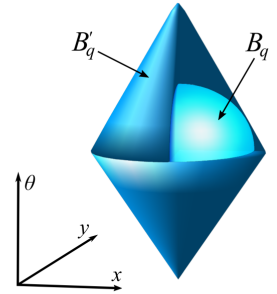

(b)

Figura 5. Nova bolha $B(q)$ inscrita na região $B^{\prime}(q)$ para um robô manipulador com três juntas(a); robô sobre rodas com acionamento diferencial (b).

Como pode ser observado na Figura 5, a bolha definida por $B^{\prime}(q)$ representa uma $n$-bola, com a área de sua superfície definida como uma $\left(n\right.$-1)-esfera. $B^{\prime}(q)$ representa 
uma região conservativa, e a nova bolha $B(q)$ é uma região, consequentemente, mais conservativa ainda.

Através dessa nova região segura, os métodos baseados em Espuma Probabilística passam a expandir bolhas sem a necessidade da representação explícita da região de obstáculos no espaço de configurações $\mathcal{C}_{\text {obs }}$.

\section{Melhoramento dos caminhos}

O PFM retorna um rosário formado por uma sequência de bolhas sobrepostas com grau de parentesco direto. Dentre os infinitos caminhos contidos no rosário, é possível extrair facilmente um caminho interligando os centros das bolhas do rosário por segmentos de reta, como pode ser observado na Figura 6.

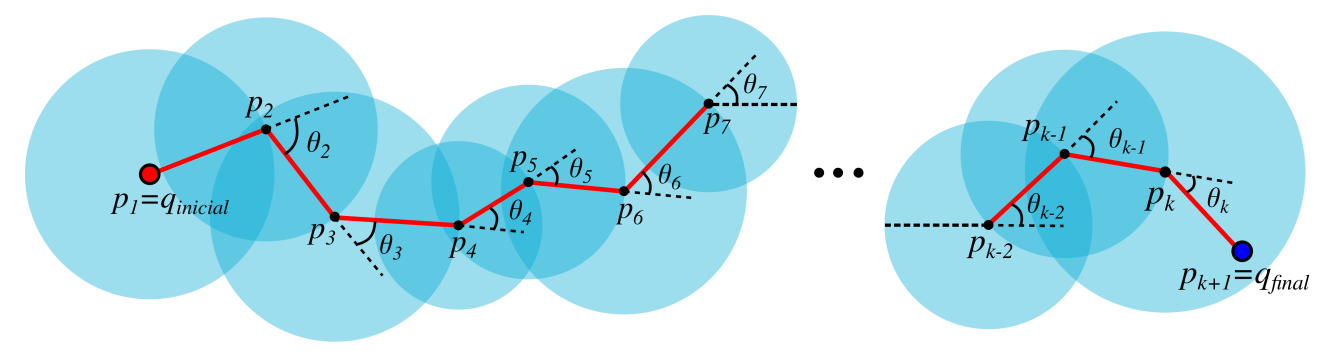

Figura 6. Rosário extraído de uma espuma, com um caminho (linhas vermelhas) partindo da configuração $q_{\text {inicial }}$ (ponto vermelho) até $q_{\text {final }}$ (ponto azul).

Considere o ponto $p_{i}$, representando a configuração localizada no centro da $i$ ésima bolha do rosário. Assim, o ponto $p_{1}$ corresponde à configuração $q_{\text {inicial }}$ e o ponto $p_{k+1}$ correspondente à configuração final $q_{\text {final }}$, sendo $k$ o número de bolhas do rosário. A partir dessa estrutura, algumas métricas capazes de avaliar o caminho podem ser definidas.

\subsection{Avaliação do comprimento do caminho}

A métrica de comprimento do caminho, avalia o seu comprimento em relação à menor distância entre as configurações $q_{\text {inicial }}$ e $q_{\text {final }}$. Proposta por [Bhowmick et al. 2017], a métrica $T C$ (taxa de comprimento do caminho) é definida por:

$$
T C=\frac{D_{e}}{D_{\text {cam }}}
$$

onde $D_{e}$ é a distância, de acordo com a métrica adotada em $\mathcal{C}$, entre as configuração inicial $q_{\text {inicial }}$ (denotado na Figura 6 pelo ponto $p_{1}$ ) e a configuração final $q_{\text {final }}$ (denotado pelo ponto $\left.p_{k+1}\right)$. Em outras palavras, $D_{e}$ representa o menor caminho possível entre os dois pontos. Considerando que um segmento de reta entre dois pontos representa o menor caminho entre eles, $T C$ representa a taxa de quão próximo o comprimento do caminho obtido $D_{\text {cam }}$ está do caminho ideal (isto é, aquele para o qual $T C=1$ ).

\subsection{Avaliação da suavidade do caminho}

A métrica de suavidade é baseada no fato de que, um caminho será mais suave em relação a outro, se a média e a variância de todos os ângulos $\theta_{i}$ (ver Figura 6) forem próximos 
de zero. A métrica $S V$ pode ser calculada por meio do estimador Erro Quadrático Médio (Mean Square Error - MSE), e pode ser expressa pela equação 7.

$$
S V=\frac{1}{k-1} \sum_{i=2}^{k} \theta_{i}^{2}
$$

onde $\theta_{i}$ é o ângulo suplementar ao ângulo de abertura, formado entre os segmentos de linha $\overline{p_{i-1} p_{i}}$ e $\overline{p_{i} p_{i+1}}$, e o valor $k-1$, que representa o número de ângulos $\theta$ a serem analisados, formados pelo rosário com um total de $k$ bolhas.

Assim, a métrica $S V$ mede a qualidade de um caminho em termos de suavidade, sendo que, o caminho mais suave possível é aquele em que os ângulos de abertura entre segmentos consecutivos formam $180^{\circ}$, e seus ângulos suplementares $\theta$ são zero $\left(\theta_{i}=\right.$ $0^{\circ}$ ), caracterizando uma linha reta. Dessa forma, quanto menor o valor da métrica de suavidade $S V$, mais suave será o caminho.

\subsection{Método para ajuste do rosário}

O rosário obtido pelo PFM pode apresentar bolhas de tamanhos desproporcionais, e posicionadas de maneira desorganizada, o que pode influenciar no comprimento do caminho e na suavidade. Dessa forma, é proposta uma estratégia baseada na métrica $T C$, para ajustar as bolhas do rosário, de forma a reposicionar as bolhas para maximizar a taxa $T C$.

Dessa forma, considere o ponto $p_{i}=\left[x_{1}^{i}, x_{2}^{i}, x_{3}^{i}, \cdots, x_{n}^{i}\right]^{T}$ sendo o centro da bolha, onde $n$ é a dimensão do espaço de configurações. É possível encontrar os melhores valores para cada ponto $p_{i}(2 \leq i \leq k)$ dentro de um rosário, ajustando cada coordenada $x_{j}^{i}(1 \leq j \leq n)$, por meio do gradiente positivo da métrica $T C$, isto é:

$$
x_{j}^{i}=x_{j}^{i}+\eta \frac{\partial T C}{\partial x_{j}^{i}}, \text { para } 0<\eta \leq 1
$$

A cada ajuste na bolha com centro $p_{i}$, seu raio é ajustado para manter sua bolha filha $p_{i+1}$ em sua superfície. Da mesma maneira, a bolha mãe $p_{i-1}$ possui seu raio ajustado para que sua bolha filha (bolha $i$ ) se mantenha em sua superfície. Além disso, a bolha ajustada deve se manter totalmente na região livre, respeitando a condição de raio mínimo.

\subsection{Métodos de suavização do caminho}

A suavidade é um aspecto de qualidade de extrema importância a ser considerado em um caminho. Para a tese, um caminho suavizado é o caminho composto por segmentos de linha sem mudanças bruscas de direção e sem curvas acentuadas.

O problema da suavização foi modelado como um problema de otimização, através do ajuste dos vértices do caminho, a fim de aumentar seus ângulos de abertura, diminuindo, consequentemente, seus ângulos suplementares $\theta$. Assim, é proposta uma estratégia para minimizar o valor de $S V$, aumentando-se assim a suavidade do caminho.

Considere um ponto $p_{i}$, vértice de um caminho. É possível encontrar os melhores valores para cada ponto $p_{i}(2 \leq i \leq k)$ dentro de um rosário, ajustando cada coordenada $x_{j}^{i}(1 \leq j \leq n)$, de acordo com o gradiente negativo da métrica $S V$, isto é: 


$$
x_{j}^{i}=x_{j}^{i}-\eta \frac{\partial S V}{\partial x_{j}^{i}}
$$

sendo $\eta$ a constante de ajuste, tal que $0<\eta \leq 1$.

\section{Resultados}

\subsection{Planejamento dos movimentos para o exoesqueleto}

Nos experimentos, foi considerado o movimento da perna em fase de balanço, com os ângulos das juntas do quadril $\theta_{h}$ e do joelho $\theta_{k}$. Além disso, o deslocamento horizontal do quadril foi considerado como variável de configuração, e para isso, foi realizada uma normalização para que o deslocamento (dado em metro) apresentasse um valor angular correspondente em radianos. Assim, uma configuração é dada pelo vetor $q=\left[\begin{array}{lll}\theta_{h} & \theta_{k} & \theta_{q}\end{array}\right]^{T}$.

Foi definido que $\theta_{q}$ parte de $\theta_{q}=0 \mathrm{rad}$, até $\theta_{q}=\frac{\pi}{2} \mathrm{rad}$, caracterizando o deslocamento total. Foi considerado nos experimentos um obstáculo com $0,5 \mathrm{~m} \mathrm{X} \mathrm{0,2}$ m X 0,15 m. A configuração inicial é dada por $q_{\text {inicial }}=\left[\begin{array}{lll}0,2 & 0,3 & 0,08\end{array}\right]^{T}$ rad e a configuração final dada por $q_{\text {final }}=\left[\begin{array}{lll}0,45 & 0,4536 & 1,45\end{array}\right]^{T}$ rad, apresentadas na Figura 7.

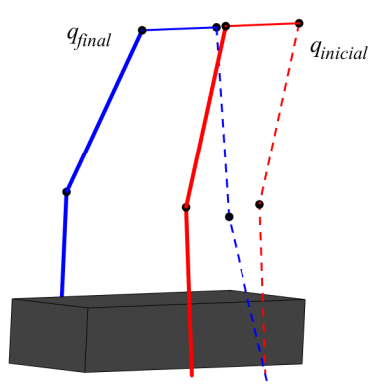

(a)

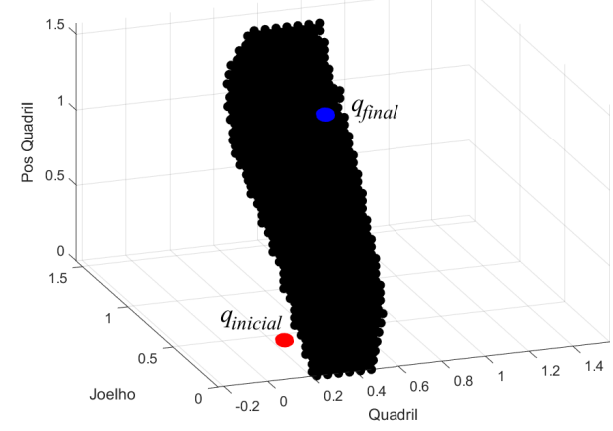

(b)

Figura 7. Posições $q_{\text {inicial }}$ e $q_{\text {final }}$ da perna para transpor obstáculo. (a) Posição no espaço de trabalho, (b) Posição mapeada para C-space.

O PFM foi configurado com o parâmetro $r_{\min }=0,045$. O valor da constante $K$ foi definido como $K=6$, dada a dimensão do espaço $n=3$ [Nascimento et al. 2021]. Para os algoritmos de Ajuste do rosário e Suavização do caminho, o parâmetro $\eta$ foi definido como $\eta=0,1$ e $\eta=0,005$, respectivamente, obtidos empiricamente, por meio de sucessivos experimentos. Os resultados para essa tarefa são apresentados na Figura 8.

A Figura 8a apresenta a espuma gerada e a Figura 8b apresenta o rosário extraído da espuma. É importante observar que as regiões de obstáculos (em preto) foram representadas apenas para ilustrar, já que a nova bolha não precisa do cálculo dessa região. A Figura 8c apresenta o rosário após aplicação do ajuste. Na Figura 8d pode-se observar o caminho $\mathcal{T}$ (linha vermelha), extraído do rosário original; o caminho $\mathcal{T}_{C A}$ (linha azul), obtido da suavização de $\mathcal{T}$; o caminho $\mathcal{T}_{R A}$ (linha magenta), obtido do rosário ajustado, e o caminho $\mathcal{T}_{R C A}$ (linha verde), que representa o caminho $\mathcal{T}_{R A}$ após o processo de suavização. As poses com a transposição do obstáculo pelo exoesqueleto, para cada um desses caminhos, são ilustradas na Figura 9. 


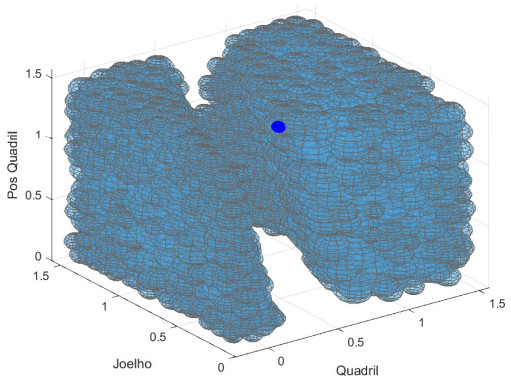

(a)

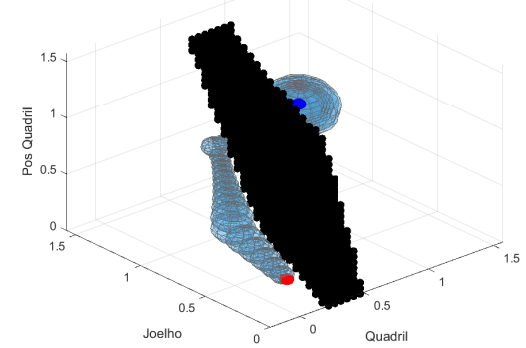

(c)

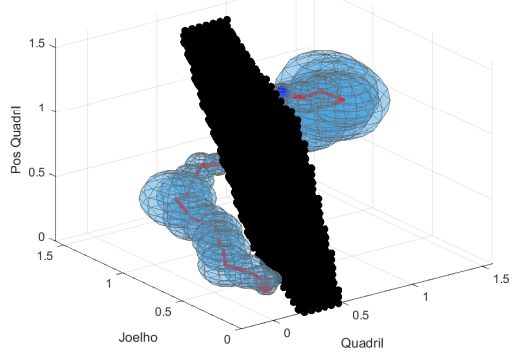

(b)

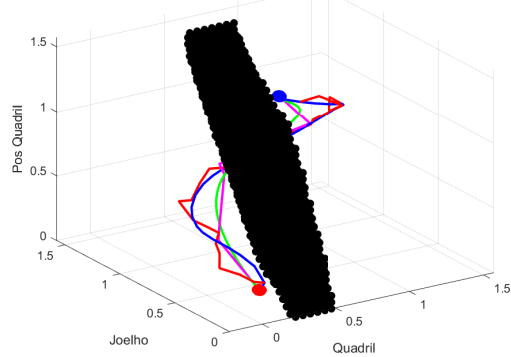

(d)

Figura 8. Resultados da transposição de obstáculos. (a) Espuma probabilística formada. (b) Rosário encontrado. (c) Rosário ajustado. (d) Caminhos $\mathcal{T}$ (vermelho), $\mathcal{T}_{C A}$ (azul), $\mathcal{T}_{R A}$ (magenta), e $\mathcal{T}_{R C A}$ (verde).

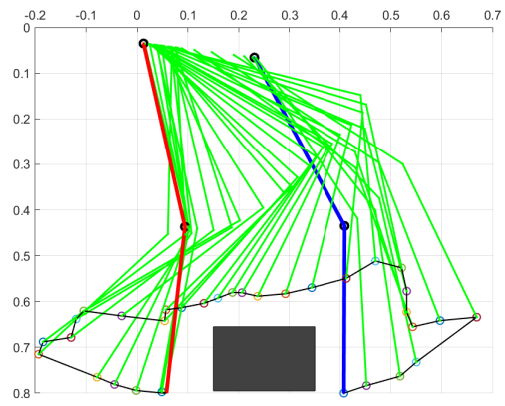

(a)

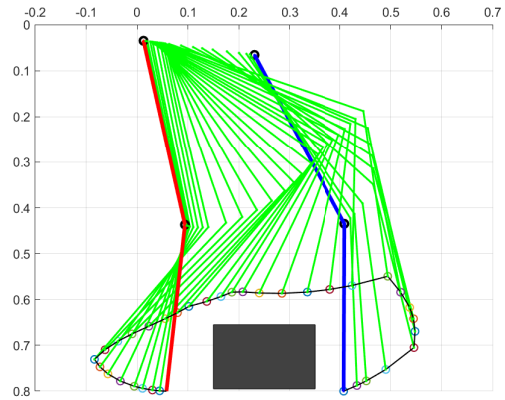

(c)

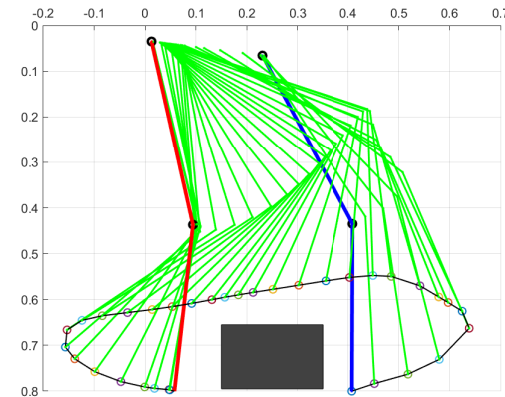

(b)

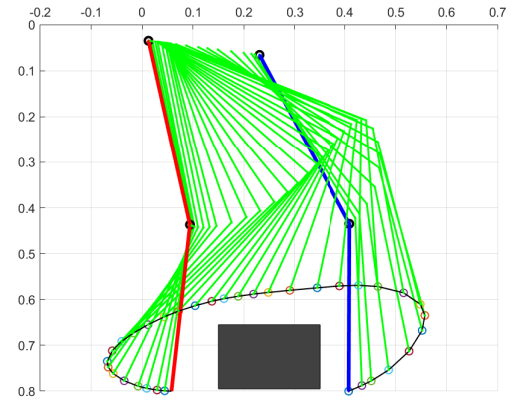

(d)

Figura 9. Poses da perna do exoesqueleto em fase de balanço, transpondo o obstáculo com os caminhos (a) $\mathcal{T}$, (b) $\mathcal{T}_{C A}$, (c) $\mathcal{T}_{R A}$, e (d) $\mathcal{T}_{R C A}$.

Para o caminho original, Figura 9a, fica evidente a necessidade da suavização. Com a aplicação da suavização no caminho original, Figura 9b, é possível perceber que o movimento resultante ainda se distancia do padrão antropomórfico. O método de ajuste 
do rosário foi capaz de encurtar mais o caminho, diminuindo o movimento, como mostra a Figura 9c. Por fim, a suavização do caminho gerado pelo rosário ajustado, proporcionou um movimento mais suave, mais curto, o que aproximou de um movimento antropomórfico, como mostra a Figura 9d. Para ilustrar o planejamento de outras tarefas, a Figura 10 apresenta as poses para as tarefas de descer e subir em um degrau, considerando o caminho original $\mathcal{T}$, e o caminho após o ajuste do rosário e sua suavização $\mathcal{T}_{R C A}$.

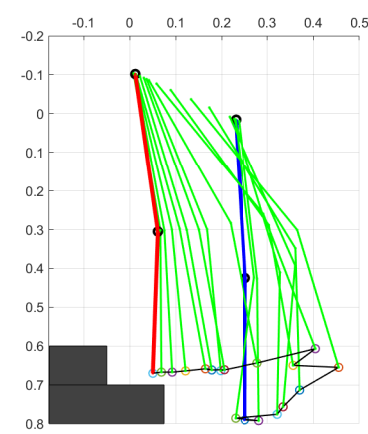

(a)

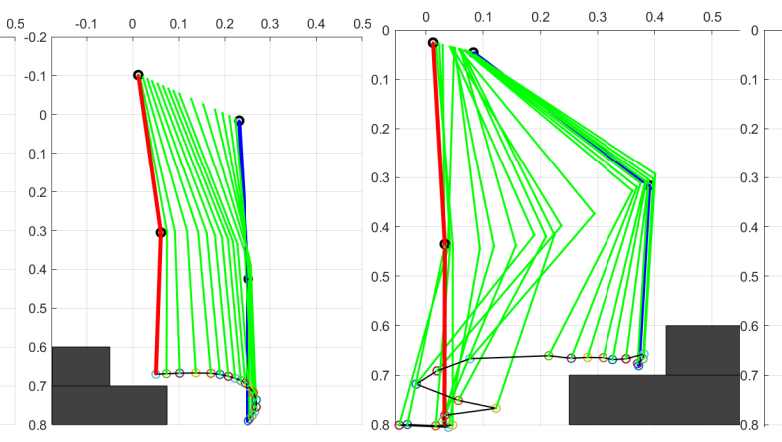

(b)

(c)

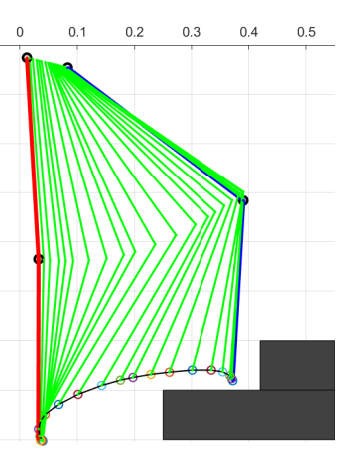

(d)

Figura 10. Poses da perna do exoesqueleto. (a) e (b) Descendo com os caminhos $\mathcal{T}$ e $\mathcal{T}_{R C A}$. (c) e (d) Subindo com os caminhos $\mathcal{T}$ e $\mathcal{T}_{R C A}$.

\subsection{Planejamento dos movimentos para o andador}

Para os experimentos com o andador inteligente CloudWalker, foram considerados dois mapas apresentados na Figura 11, com obstáculos representados no espaço de trabalho. O primeiro cenário (Figura 11a) representa um ambiente com um corredor estreito, para testar a habilidade propagação da espuma. O segundo cenário (Figura 11b) apresenta duas possíveis rotas, uma de menor comprimento, porém mais estreita, e a segunda mais longa, porém, mais ampla. Para o Mapa 1, o $r_{\text {min }}$ foi definido como $r_{\text {min }}=0,2 \mathrm{~m}$ e para o Mapa 2, $r_{\min }=0,25 \mathrm{~m}$. Para ambos mapas foi definido o parâmetro $K=6$.

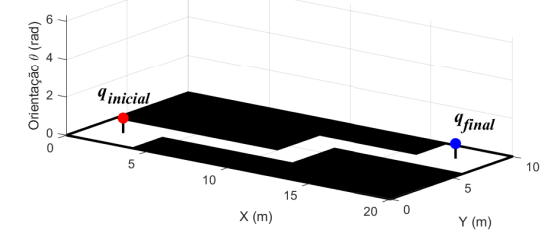

(a)

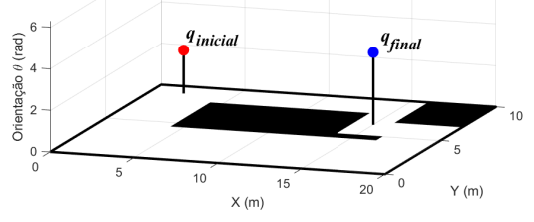

(b)

Figura 11. (a) Mapa 1 com corredor estreito. (b) Mapa 2 com duas rotas possíveis.

A Figura 12 apresenta um experimento realizado por meio do aplicação do Método da Espuma Probabilística (PFM) no Mapa 1.

O PFM gerou uma espuma com muitas bolhas, como pode ser observada na Figura $12 \mathrm{a}$, com um total de bolhas computadas de $n_{\text {bolhas }}=1544$, com tempo de busca $T=16,0517$ segundos. É possível perceber que, devido a utilização da nova bolha, não foi necessária a computação da região de obstáculos. Em relação à qualidade do caminho 
planejado, apresentado na Figura 12c, a taxa de comprimento do caminho obtido foi de $T C=0,7048$ e apresentando uma suavidade de $S V=0,8051$. O caminho obtido foi submetido ao processo de suavização, como mostram as Figuras 12d e 12e, para finalmente ser aplicado ao robô, onde o movimento é apresentado na Figura 12f.

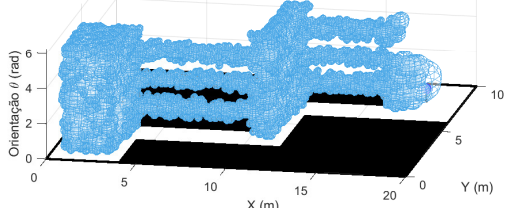

(a)

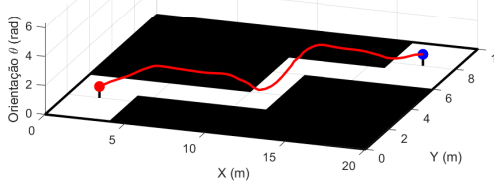

(d)

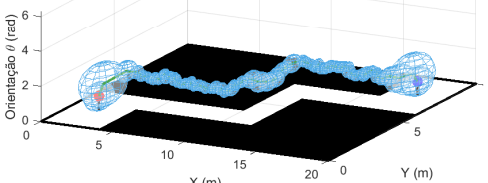

(b)

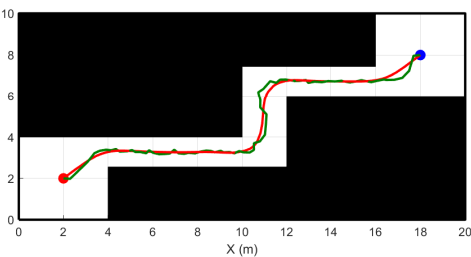

(e)

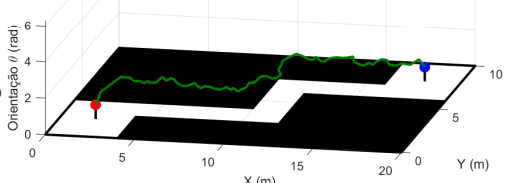

(c)

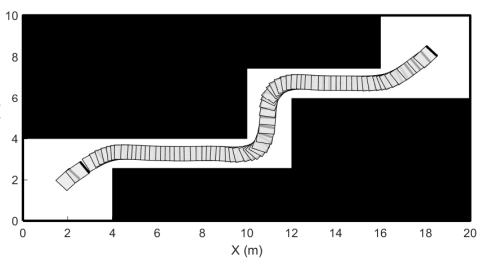

(f)

Figura 12. Planejamento para o andador com o Mapa 1. (a) Espuma formada. (b) Rosário encontrado. (c) Caminho extraído do rosário. (d) Caminho suavizado. (e) Comparação dos caminhos. (f) Movimento realizado pelo robô para o caminho suavizado.

A Figura 13 apresenta um experimento realizado por meio da aplicação do Método da Espuma Probabilística (PFM) no Mapa 2.

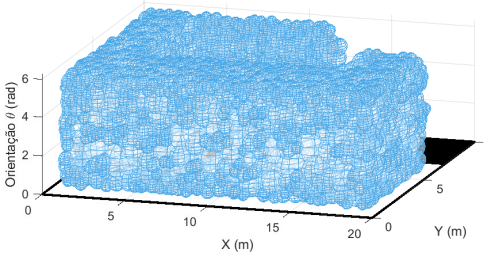

(a)

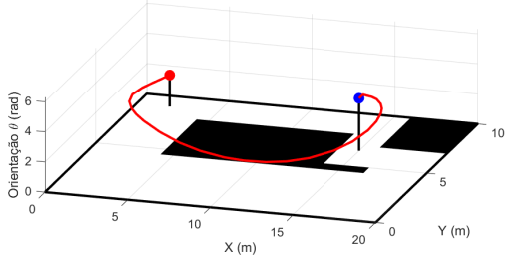

(d)

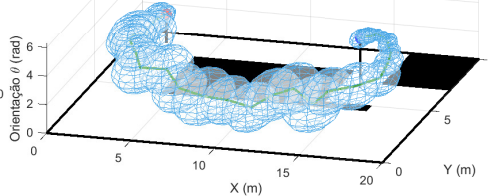

(b)

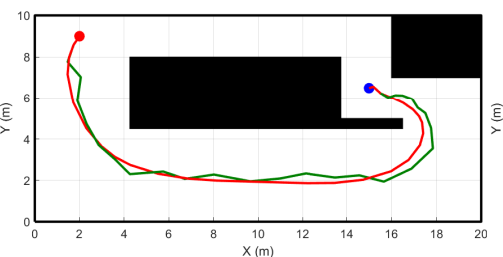

(e)

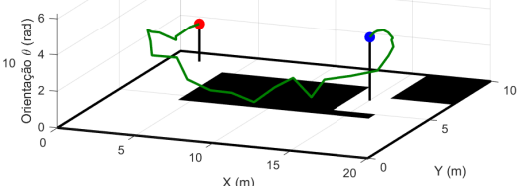

(c)

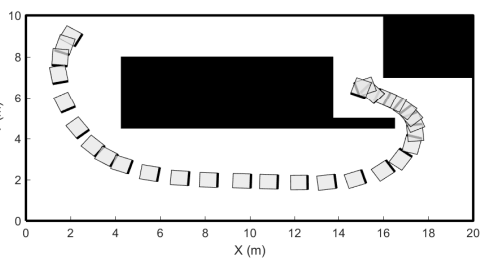

(f)

Figura 13. Planejamento com o andador para o Mapa 2. (a) Espuma formada. (b) Rosário encontrado. (c) Caminho original extraído do rosário. (d) Caminho suavizado. (e) Comparação entre os caminhos. (f) Movimento realizado pelo robô para o caminho suavizado.

É possível observar, na Figura 13a, que o PFM gerou uma espuma densa, reali- 
zando uma boa cobertura do espaço livre, totalizando 1795 bolhas, em 13,2180 segundos. O caminho obtido (Figura 13c) apresentou a taxa de comprimento de $T C=0,4220$ e a suavidade do caminho foi de $S V=0,7308$. A Figura 13d apresenta o caminho resultante do planejamento suavizado, e na Figura 13e é apresentada uma comparação com o caminho original e sua versão suavizada, e a Figura 13f ilustra a trajetória desempenhada pelo robô com o caminho suave.

\section{Conclusões}

Esse trabalho apresentou, de forma resumida, alguns resultados desenvolvidos durante o doutorado, envolvendo o aprimoramento do método da Espuma Probabilística, aplicado no planejamento de caminho para dois robôs assistivos. Por meio das abordagens desenvolvidas, o método foi capaz de planejar caminhos seguros, sem a necessidade da computação da região de obstáculos no espaço de configurações. Além disso, por meio de estratégias de aprimoramento do caminho desenvolvidas para esse método de planejamento, foi possibilitado movimentos mais antropomórficos para o exoesqueleto.

\section{Referências}

Araujo, M. V., Alsina, P. J., Roza, V. C. C., and Melo, N. B. (2015). Powered Orthosis Ortholeg: Design and Development. IEEE Latin America Transactions, 13(1):90-95.

Berglund, T., Brodnik, A., Jonsson, H., Staffanson, M., and Soderkvist, I. (2009). Planning smooth and obstacle-avoiding b-spline paths for autonomous mining vehicles. IEEE Transactions on Automation Science and Engineering, 7(1):167-172.

Bhowmick, S., Mukhopadhyay, J., and Deb, A. K. (2017). Fast path planning on planar occupancy grid exploiting geometry of obstacles. In 2017 Ninth International Conference on Advances in Pattern Recognition (ICAPR), pages 1-6.

Nascimento, L. B., Barrios-Aranibar, D., Santos, V. G., Pereira, D. S., Ribeiro, W. C., and Alsina, P. J. (2021). Safe path planning algorithms for mobile robots based on probabilistic foam. Sensors, 21(12):4156.

Plaku, E., Plaku, E., and Simari, P. (2018). Clearance-driven motion planning for mobile robots with differential constraints. Robotica, 36(7):971-993.

Quinlan, S. (1995). Real-Time Modification of Collision-Free Paths. Doctoral Dissertation, Stanford University Stanford, CA.

Scheidegger, W. M., de Mello, R. C., Sierra M., S. D., Jimenez, M. F., Múnera, M. C., Cifuentes, C. A., and Frizera-Neto, A. (2019). A novel multimodal cognitive interaction for walker-assisted rehabilitation therapies. In 2019 IEEE 16th International Conference on Rehabilitation Robotics (ICORR), pages 905-910.

Silveira, Y. S. and Alsina, P. J. (2016). A New Robot Path Planning Method Based on Probabilistic Foam. In 2016 XIII Latin American Robotics Symposium and IV Brazilian Robotics Symposium (LARS/SBR), pages 217-222.

Volna, E. and Kotyrba, M. (2018). Pathfinding in a dynamically changing environment. In Nguyen, N. T., Hoang, D. H., Hong, T.-P., Pham, H., and Trawiński, B., editors, Intelligent Information and Database Systems, pages 265-274, Cham. Springer International Publishing. 